\title{
Instruments for cognitive screening in the elderly
}

\begin{abstract}
Aging is a natural process that produces a progressive decrease in the functional and cognitive capacity of people, also known as senescence. However, overloads from events such as illness, accidents and emotional stress can cause a pathological condition that will require assistance. One of the conditions that can affect the elderly are cognitive deficits. Cognitive assessment is an important process in this context because it measures the measure of an individual's ability to acquire and use information in order to adapt to the demands of the environment. There are for this purpose several instruments for cognitive screening that configure scales of easy and fast application, comprising the domains: temporo-spatial orientation, immediate memory and evocation, calculation, language, naming, comprehension, writing, praxia, among others. The cognitive battery is a set of several combined and correlated screening instruments. The impact of cognitive evaluation on the health of the elderly presupposes that a cognitive-functional evaluation is essential for obtaining a broad diagnosis, which will both serve preventive actions as well as to assist in the treatment decisions and care needed for elder care. The purpose of this article is to present the most used cognitive evaluation instruments for cognitive screening of the elderly, as well as the parameters used in these evaluations.
\end{abstract}

Volume 3 Issue 4 - 2017

\author{
Juliana Balbinot Reis Girondi,' Maria \\ Aparecida Griza, ${ }^{2}$ Luciara Fabiane Sebold, ${ }^{3}$ \\ Jordelina Schier, ${ }^{4}$ Karina Silveira de Almeida \\ Hammerchmidt \\ 'Department of Nursing, Federal University of Santa Catarina, \\ Brazil \\ ${ }^{2}$ State Secretary of Health of Santa Catarina, Brazil \\ ${ }^{3}$ Department of Nursing, Federal University of Santa Catarina, \\ Brazil \\ ${ }^{4}$ Coordinator of the nucleus of studies of the third age, Federal \\ University of Santa Catarina, Brazil
}

\section{Correspondence: Juliana Balbinot Reis Girondi, Department of Nursing, Federal University of Santa Catarina, Brazil, Tel} 5548372I-3457, Email juliana.balbinot@ufsc.br

Received: June 15, 2017 | Published: August 21, 2017

Keywords: elderly, cognitive evaluation, cognitive deficit assessment, cognitive screening scale

\section{Introduction}

Population aging, and in particular the aging of older people, has been gaining increasing importance in recent years as a result of the proportionately increasing number of older people compared to the number of people of other age groups. The decline in fertility and the increase in life expectancy are factors that explain this phenomenon, which is more clearly observed in developed countries, but also in developing countries. These demographic changes are reflected in the epidemiological profile, which shows, among other consequences, the increase in no communicable chronic diseases (NCDs), which, when they are not fatal, frequently lead to partial or total dependence of the individual, with serious repercussions for him, his family and society.

The minimization of the consequences of these negative events, is in the early treatment, being this preponderant factor in the rehabilitation; Is the broad knowledge of the individual conditions of the elderly, evaluated in all aspects, including the physiological, cognitive, psychological, socioeconomic and environmental. The importance of this broad vision stems from the fact that being an elderly patient, the aging process itself may be promoting changes in the mentioned aspects, which may become restrictive factors to an effective rehabilitation therapeutic plan.

Accurate measurement of cognitive status is an emerging challenge, and it is necessary to identify the non-apparent weaknesses, the potential prognosis and adequately delineate the treatment behaviors. From the specific point of view of cognitive functions, problem-solving ability decreases with age, since it encompasses many cognitive processes, from low level (perception and recognition) to high level (memory, reasoning and decision making). ${ }^{1}$ Cognitive deficits can influence the recovery of the patient's functional status during rehabilitation, causing this process to extend, making treatment difficult and compromising patients' quality of life. ${ }^{2}$
The presence of disturbances in cognition is an important complication of recovery, directly affecting the process of rehabilitation and recovery of the patient. ${ }^{3}$ Cognitive dysfunctions may interfere with an individual's ability to understand precisely all parts of their condition, since a cognitively impaired patient may have difficulty keeping a memory of past events and associating related information. ${ }^{4}$ Cognitive screening instruments are quick and easy to perform tests that provide approximate characterization of various cognitive functions and are indicated for use in distinguishing between normal and pathological conditions. ${ }^{5}$

Cognitive assessment is the measure of an individual's ability to acquire and use information in order to adapt to the demands of the environment. On the other hand, the cognitive tracing instrument is a scale of easy and quick application, comprising the domains: temporospatial orientation, immediate memory and recall, calculation, language, naming, comprehension, writing, praxia, among others. The cognitive battery is a set of several combined and correlated screening instruments. In this sense, the evaluation can contribute to an increase in diagnostic accuracy, a decrease in hospital admissions, a decrease in the use of medication and in the reduction of expenses, thus constituting an important tool for planning public Health for the population.

\section{Discussion}

This is an Integrative Review of literature aiming to identify in the scientific literature studies that show the cognitive screening instruments used in the evaluation of the elderly. The search strategies were listed: organization of descriptors or keywords according to the differentiation between databases; Combination of two search terms (Descriptors in Health Sciences - DeCS/MeSH: cognitive evaluation, cognitive deficit assessment, cognitive and elderly screening scale); Combination of Boolean terms (AND, OR). We used the electronic 
bibliographic research in the databases: Medical Literature Analysis and Retrieval System Online (MEDLINE), Latin American and Caribbean Literature in Health Sciences (LILACS), Scientific Electronic Library Online (SciELO), Spanish Bibliographic Index of Health Sciences (IBECS).

All material found was separated in the form of abstracts. Afterwards, a floating reading of the same was carried out to check their validity, according to the objective of the study. Those considered pertinent have been able to retrieve their complete texts, all of which are organized in a Excel for Windows Program worksheet, with the main information: code, title, authors, year of publication, database, type of work, objective, method and instruments Referenced. This documentary body went through an even more careful reading, in order to extract the main theme. Once the thematic survey was carried out, it was possible to categorize by instrument class. ${ }^{6}$

The data were synthesized in a descriptive way and analyzed from the thematic analysis of Bardin, allowing the reader to know the literature on the subject investigated. We selected 24 studies on 13 cognitive screening instruments that comprised the following categories: Cognitive evaluation batteries. Cognitive screening instruments; Cognitive assessment instruments, according to the caregiver. Scales for health professionals. The main instruments used and their evaluative parameters are presented in Table 1.

Table I Main instruments of cognitive screening in the elderly and evaluative parameters. Brazil, 20 I7

Instruments
Cognitive evaluation batteries
Consortium to Establish A Registry For Alzheimer's Disease
(CERAD)
Cambridge cognitive examination (CAMCOG)
Cambridge cognitive examination - Revised (CAMCOG-R)

Addenbrooke's cogntive examination - Revised (ACE-R)

Cognitive screening brief battery (BBRC)

\section{Cognitive screening instruments}

Mini-Mental state examination (MMSE)

Clock drawing test (TDR)

Blessed dementia scale (EBD)

Lawton \& brody scale

Pfeffer functional activities questionnaire (QAFP)

Montreal cognitive assessment (MoCA)

Track testing

\section{Parameters used}

Memory (fixation, evocation and recognition), language (naming and verbal fluency), constructive praxis and executive function.

Orientation, language (spontaneous speech and expression), memory, attention, concentration, praxia, perception and abstract thinking.

Language, memory, perception and executive functions (ideational fluency and visual reasoning)

Recall, anterograde, retrograde, verbal fluency, language (comprehension, repetition, naming, comprehension and writing, visual skills, perceptual skills, recall and recognition).

Executive functions, language and skills.

Temporal-spatial orientation, immediate memory and evocation, calculation, language, naming, comprehension, writing and praxia.

Executive functions, visuospatial processing and organization, perseveration and semantic knowledge

Daily Life Activities, Changing Habits, and Behavioral Disorders. Instrumental Activities of Daily Living (AIVD's).

Evaluates the performance of Instrumental Activities of Daily Living that also involve cognitive abilities.

Executive functions, visuospatial skills, naming, memory retrieval, digits, sentence, abstract reasoning and orientation.

Planning, organization, motor dexterity, operational memory, attention and concentration

\section{Cognitive evaluation instruments of the elderly, according to the caregiver}

Informant questionnaire on cognitive decline in the elderly (IQCODE)

Cognitive change questionnaire (QM22)
Memory, time-space orientation, executive functions, learning, calculation and judgment. Allows screening and diagnosis of dementia syndrome based on information reported by caregivers

Level of consciousness, eye movements, visual field, facial movements, motor function of upper limb and lower limb, limb ataxia, sensibility, language, dysarthria, spatial neglect.
Cognitive Change Questionnaire (QM22) Level of consciousness, eye movements, visual field, facial movements, motor function of upper limb and lower limb, limb ataxia, sensibility, language, dysarthria, spatial neglect. Cognitive evaluation batteries consist of a set of standardized instruments used by qualified professionals to allow the evaluation of cognitive functions in order to subsidize the detection of specific cognitive declines. Its use in the evaluation of the elderly is a valuable tool, considering that the elderly tend to have an atypical presentation when compared to younger individuals, which makes differential diagnosis a fundamental resource. 
The tests are quick application cognitive screening tools that can be used by qualified professionals. Depending on, they may be inserted in a battery or individually applied. After the elderly go through the extensive evaluation, where cognitive problems were identified, it is up to the professional to use specific scales, individually or possibly combined, to obtain a more accurate measure of their cognitive functions. ${ }^{7}$ The results indicated a predominance of Mini Mental State Examination (MMSE).

\section{Conclusion}

It should be emphasized the complexity of care for the elderly, especially those with some health vulnerability. The importance of assessing the prevalence of cognitive deficits before the onset of the acute pathology that motivates the hospitalization of the elderly at all levels of care, and then develops the integral geriatric evaluation. This assessment includes the cognitive function to detect the frail elderly.

\section{Acknowledgements}

This study is linked to the matrix project entitled "The network of health care and social support to the elderly person with disabilities in the great Florianópolis and care technologies", which was contemplated in the edict of the Foundation for Support to Research and Innovation of the State of Santa Catarina (PPSUS/FAPESC), Brazil, in 2013.

\section{Conflict of interest}

The author declares no conflicts of interest.

\section{References}

1. Gomes APP. A Intervenção Psicomotora no Envelhecimento Patológico. Lisboa: Universidade Técnica Lisboa; 2013.

2. Dantas AATSG, Torres SVS, Farias IMA, et al. Rastreio cognitivo em pacientes com acidente vascular cerebral: um estudo transversal. J Bras Psiquiatr. 2014;63(2):98-103.

3. Costa FA, Silva DLA, Rocha VM. Estado neurológico e cognição de pacientes pós-acidente vascular cerebral. Rev Esc Enferm USP São Paulo. 2011;45(5):1083-1088.

4. Kawamoto R, Yoshida O, Oka Y. Factors related to functional capacity in community-dweling elderly. Geeratrics and Gerontology International. 2004;4(2):105-110.

5. Schwanke CHA. Updates in geriatrics and gerontology IV: demographic, psychosocial and clinical aspects of aging. EdiPUCRS, Porto Alegre: Springer; 2012.

6. Freitas S, Alves L, Simões MR, et al. Importância do rastreio cognitivo na população idosa. Revista Eletrônica de Psicologia. 2013;3(1):4-22.

7. Ministério da Saúde. Secretaria de Atenção à Saúde. Departamento de Atenção Básica. Envelhecimento e saúde da pessoa idosa. Cadernos de Atenção Básica. Brasil: Springer; 2006. 192 p. 